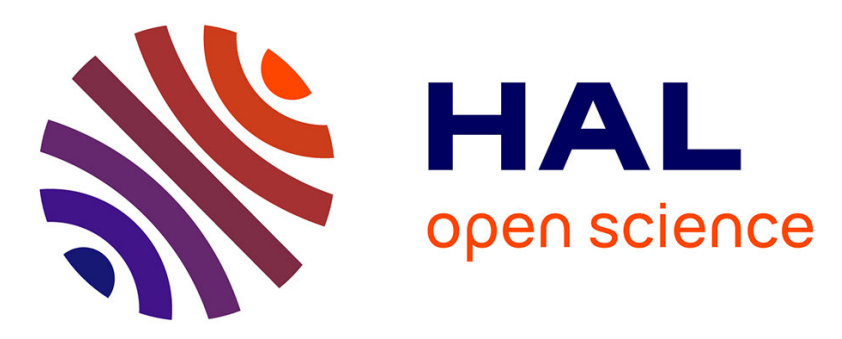

\title{
Multi-objective Trajectory Optimization to Improve Ergonomics in Human Motion
}

Waldez Gomes, Pauline Maurice, Elö̈se Dalin, Jean-Baptiste Mouret, Serena Ivaldi

\section{- To cite this version:}

Waldez Gomes, Pauline Maurice, Eloïse Dalin, Jean-Baptiste Mouret, Serena Ivaldi. Multi-objective Trajectory Optimization to Improve Ergonomics in Human Motion. IEEE Robotics and Automation Letters, 2022, 7 (1), pp.342 - 349. 10.1109/LRA.2021.3125058 . hal-03281827v3

\section{HAL Id: hal-03281827 https://hal.science/hal-03281827v3}

Submitted on 22 Dec 2021

HAL is a multi-disciplinary open access archive for the deposit and dissemination of scientific research documents, whether they are published or not. The documents may come from teaching and research institutions in France or abroad, or from public or private research centers.
L'archive ouverte pluridisciplinaire HAL, est destinée au dépôt et à la diffusion de documents scientifiques de niveau recherche, publiés ou non, émanant des établissements d'enseignement et de recherche français ou étrangers, des laboratoires publics ou privés. 


\title{
Multi-objective Trajectory Optimization to Improve Ergonomics in Human Motion
}

\author{
Waldez Gomes ${ }^{1}$, Pauline Maurice ${ }^{1}$, Elö̈se Dalin ${ }^{1}$, Jean-Baptiste Mouret $^{1}$, Serena Ivaldi ${ }^{1}$
}

\begin{abstract}
Work-related musculoskeletal disorders are a major health issue often caused by awkward postures. Identifying and recommending more ergonomic body postures requires optimizing the worker's motion with respect to ergonomics criteria based on the human kinematic/kinetic state. However, many ergonomics scores assess different risks at different places of the human body, and therefore, optimizing for only one score might lead to postures that are either inefficient or that transfer the risk to a different location. We verified, in two work activities, that optimizing for a single ergonomics score may lead to motions that degrade scores other than the optimized one. To address this problem, we propose a multi-objective optimization approach that can find better Pareto-optimal tradeoff motions that simultaneously optimize multiple scores. Our simulation-based approach is also user-specific and can be used to recommend ergonomic postures to workers with different body morphologies. Additionally, it can be used to generate ergonomic reference trajectories for robot controllers in humanrobot collaboration.
\end{abstract}

Index Terms-Human Factors and Human-in-the-Loop; Human and Humanoid Motion Analysis and Synthesis; Modeling and Simulating Humans

\section{INTRODUCTION}

W ORK-RELATED musculoskeletal disorders (WMSDs) are among the first causes of occupational diseases worldwide, representing a major health issue, with important costs for companies and society. They develop when biomechanical demands repeatedly exceed the workers' physical capacities, and, along with force exertion, awkward postures represent one of their major risk factors [1]. In many situations, workers are able to choose among a variety of postural strategies to execute a task. Yet, their natural choice does not always match the best strategy with respect to long-term health. For instance, several studies reported that novice workers can adopt strategies that result in higher biomechanical loading in comparison to experienced workers [2]. Recommending better ergonomic postures for specific tasks is, therefore, a promising avenue to help to reduce WMSDs among workers.

Posture recommendation requires a prior identification of the best postural strategy for each task -taking into account

Manuscript received: June, 8, 2021; Revised August, 26, 2021; Accepted October, 27, 2021.

This paper was recommended for publication by Editor Jee-Hwan Ryu upon evaluation of the Associate Editor and Reviewers' comments. This work was partially supported by European Union's H2020 Research and Innovation Programme under Grant Agreement No. 731540 (Project AnDy), and by French Research Agency (ANR) under Grant No. ANR-20-CE33-0004 (project ROOIBOS).

${ }^{1}$ The authors are with Université de Lorraine, CNRS, Inria, LORIA, F54000 Nancy, France. waldez.azevedo-gomes-junior@inria.fr, surname.name (at) inria.fr

Digital Object Identifier (DOI): see top of this page.

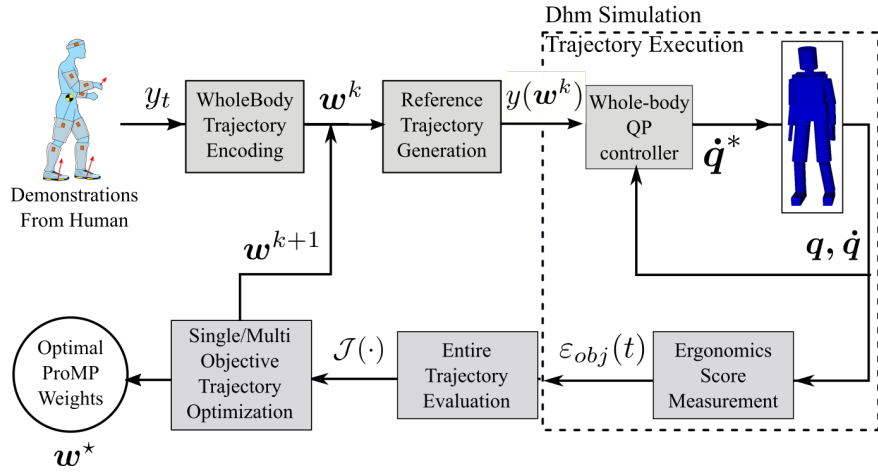

Fig. 1: Ergonomics human motion optimization. The entire motion is encoded into motion primitives that can be readily optimized with respect to a single, or multiple, ergonomics scores using a user-specific Digital Human Model (DHM) Simulation for motion evaluation.

workplace constraints- adapted to the specific individual. The best strategy usually depends on individual factors, such as body morphology, or joint capacities. The question of identifying ergonomic postural strategies is also pushed forward by the growing interest for collaborative robotics as a potential solution to improve work conditions in industry. In addition to the direct physical assistance -such as weight compensation or force amplification- that they can provide, collaborative robots can be used to drive workers towards ergonomic postures that minimize the risks of WMSDs [3]-[6]. This can be achieved, for instance, through the positioning of the robot end-effector that constrains the human hand position, which in turn influences the human posture (assuming an interaction at the human hand/robot end-effector [7]). However, such assistance requires selecting a robot end-effector trajectory that corresponds to an ergonomic human posture. Such a posture needs to be determined beforehand.

State-of-the-art human-robot applications that improve the human partner's ergonomics usually take in consideration only one ergonomics score [4]-[6], [8]. However, single-objective optimization may not be sufficient to obtain ergonomically adequate whole-body motions, since optimizing for only one criterion often produces motions that are less ergonomic in other body regions; e.g., minimizing only the back flexion ignores the leg motion or efforts at the shoulder joints.

In this paper, we propose to use multi-objective optimization to generate several Pareto-optimal motions that simultaneously optimize different ergonomics scores. A simulationbased optimization approach generates ergonomic whole-body 
motions for different body morphologies and activities (Fig. 1). Initial demonstrations from motion capture are used as feasible motions to warm-start the optimization process. The motions are parameterized by probabilistic movement primitives (ProMPs), that can encode several task demonstrations. A user-specific Digital Human Model (DHM) simulates the whole-body motion in a physics engine, and the simulation is used to estimate several ergonomics scores based not only on the body's posture, but also on its joint torques. A multiobjective optimization algorithm (NSGA-II [9]) is used to generate several possible Pareto-optimal solutions (i.e., wholebody motions) that represent trade-offs among the different ergonomics criteria. This approach is user- and activity-specific, and generates a variety of different movements that promotes the different ergonomics criteria, producing ergonomically reasonable motions for the DHM.

After describing the method in detail (section III), we empirically show that: 1) ergonomics optimization must be user-specific (experiment 1 in section $[\mathrm{IV} / \mathrm{V}$; 2) optimizing for one single criteria may lead to non-ergonomic motions for other criteria, which means ergonomics criteria can conflict (experiment 2 in section $[\mathrm{IV} / \mathrm{V}$; 3 ) optimizing simultaneously for several criteria using multi-objective optimization leads to a set of motions that are more adequate w.r.t. ergonomics.

\section{RELATED WORK}

Prior work used human models to automate whole-body motion analysis for a given activity [10], [11]. There is a recent trend in the human-robot interaction community to use them to improve the human posture with respect to ergonomics scores during physical interactions. For instance, Marin et al. optimized a shared object's position in order to minimize the maximum muscle activation signal taken from a fast-tocompute musculoskeletal surrogate model [5]. Van der Spaa et al. optimized a discrete sequential plan of poses for a shared object during its transportation by both human and robot, with respect to the Rapid Entire Body Assessment (REBA) score, a standard whole-body ergonomics score [12].

Other works continuously evaluated the human kinematics/kinetics to try to influence the human posture with different robot actions. Shafti. et al. used wearable sensors to compute the Rapid Upper-Limb Assessment (RULA) score and adapt the robot's end-effector accordingly until the ergonomics evaluation was considered satisfactory [6]. Kim et al. minimized the human joint torque due to an external load [4]. Similar optimization techniques were used to improve human operator ergonomics during teleoperation [13], [14].

The examples above considered single ergonomics scores. However, given the multi-factorial causes of WMSDs, optimizing the movement for one ergonomics score could deteriorate other possible antagonistic scores. For this matter, there are some examples of multi-objective ergonomics optimization in the literature. For instance, Xiang et al. optimized a human's posture w.r.t. ergonomics and stability scores [15], and Iriondo et al. optimized a workstation setup parameter w.r.t RULA, and the human's upper-arm elevation angle [16]. In a physical human-robot application, Maurice et al. optimized a

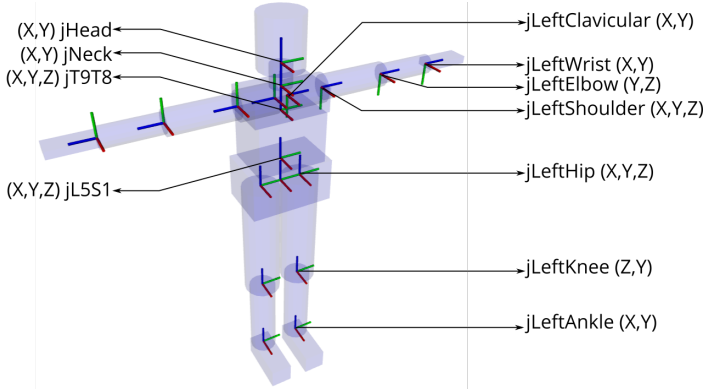

Fig. 2: Digital Human Model (DHM) joints description. The axes are $\mathrm{X}=$ Red, $\mathrm{Y}=$ Green, $\mathrm{Z}=$ Blue.

robot's design parameters to simultaneously improve multiple ergonomics scores [17]. Figueredo et al. combined muscle activation predictions and the REBA score to calculate a comfortability index that can be used in a physical humanrobot interaction to guide the human partner towards postures that minimize both types of scores [18].

In this work, we propose an approach to optimize user- and activity-specific whole-body motions with respect to multiple ergonomics scores. We also show that the resulting optimal motions are sensitive to different body morphologies and ergonomics scores. A major advantage of our approach is that we do not aggregate the different ergonomics criteria in a weighted sum, but instead we run a truly multi-objective optimization, i.e. we generate a Pareto front of non-dominated solutions [9]. Thereby, we do not preset weights for each criterion prior to the optimization, which would likely be activity or even user-specific (e.g., in case of a known user disability or pathology) and require the expertise of an ergonomist.

\section{METHODS}

\section{A. Digital Human Model Simulation}

The DHM used in this work (Fig. 2p consists of 19 rigid bodies linked together by 18 compound joints, for a total of 43 DoFs (11 for the back and neck, 9 for each arm including the sternoclavicular joint, and 7 for each leg), plus 6 DoFs for the free-floating base. Each DoF is a revolute joint controlled by a single actuator. Different human morphologies are easily generated from a desired body mass and height, by scaling the geometric and inertial parameters of the human model according to average anthropometric coefficients [19], [20].

The DHM is simulated in a physics engine (Dynamic Animation and Robotics Toolkit, DART [21]) and controlled by a multi-task quadratic programming $(\mathrm{QP})$ controller [22] that generates the motion that will be evaluated. The $\mathrm{QP}$ controller takes reference Cartesian trajectories that define the activity as input, and outputs desired joint velocities for the DHM. The QP is set to minimize the tracking error of these references while handling task priorities defined by the user. The priorities are defined by hierarchical levels, and tasks within the same level are further prioritized by their task weights. At the first priority level of the QP controller, there is a fixed Cartesian task for both feet that keeps the DHM in double support, and a task for the DHM's center of mass, to balance the DHM. At the second priority level, there are 
Cartesian tasks for the hands, pelvis, and head, as well as a task that defines reference body postures. Here, the weights of all tasks are defined according to the work activity.

\section{B. Ergonomics Evaluation}

To obtain ergonomic motions from the optimization, we need to define ergonomics scores as objective functions to our formulation. There are many possible ergonomics scores typically representing different physiological phenomena that could increase the risk of developing WMSDs [23]. However, there is no strict consensus on a single score to use for motion optimization. Moreover, they might be antagonistic among themselves, e.g. the same movement could produce results that are ergonomically suitable for a score, and not for another.

For this reason, instead of using an aggregated score, that is often activity-specific, we consider several scores, $\varepsilon_{o b j}$, separately (Tab. I). In order to obtain an evaluation of the entire trajectory execution, we use a cost proportional to the squared RMS value of $\varepsilon_{o b j}$ for each score, for the entire activity duration:

$$
\mathcal{J}_{o b j}=\sum_{t \in[0 \ldots T]} \varepsilon_{o b j}^{2}(t)
$$

where $T \in \mathbb{R}$ is the final simulation instant. Below, we describe each of the selected scores $\varepsilon_{o b j}$.

RULA-C or RULA Continuous: The Rapid Upper Limb Assessment (RULA) tool [24] is often used by ergonomists to evaluate work activities involving upper-body motion. It consists of a score ranging from 1 to 7 , calculated based on the joint positions, the force/load applied at the worker's arm, and how many times the activity is repeated. RULA time evolution during a work activity is likely to have discontinuities, and plateaus that make its domain exploration less efficient for many optimizers. To alleviate this problem, we propose a continuous version of RULA instead: RULA-C, $\varepsilon_{r c} \in \mathbb{R}^{+}$. To compute RULA-C, we fit second-degree polynomial functions to calculate intermediate scores for the RULA joints. The joint scores for each limb are combined with weighted sums whose weights are computed from linear regressions of the standard RULA tables. Moreover, differently from RULA, RULA-C only takes into account the body posture.

Normalized whole-body Effort: The torques at every joint are summed to quantify the whole-body effort (table I), where all joint torques are normalized w.r.t. average maximum human capacity [19] in order to handle the joint torque capabilities.

Local measurements: WMSDs at the shoulder and lumbar areas are among the most common in the population [1], therefore, we chose scores that target them. For the shoulder joint, we monitor its absolute torque values, $\varepsilon_{t s h}$, and for the lumbar joint, we monitor its absolute torque values, $\varepsilon_{t l b}$, and the back flexion angle, $\varepsilon_{\text {back }}$.

\section{Whole-body Trajectory Parameterization}

The reference trajectories to the QP controller define the whole-body movement, from which, a few of them are selected to be optimized, and for this reason, parameterized by Probabilistic Movement Primitives (ProMPs), which can represent
TABLE I: Ergonomic Evaluation Scores. $\varepsilon_{o b j}(t)$ is the instantaneous score.

\begin{tabular}{c|c|c} 
Description & Score & $\varepsilon_{\mathrm{obj}}(t)$ \\
\hline RULA-C & Regression of RULA $\mid \overline{24}$ & $\varepsilon_{\mathrm{rc}}$ \\
\hline $\begin{array}{c}\text { Normalized } \\
\text { whole-body Effort }\end{array}$ & $\frac{1}{n_{\text {joints }}} \sum_{\text {iejoints }}\left(\frac{\tau_{t}^{i}}{\tau_{\text {max }}^{i}}\right)^{2}$ & $\varepsilon_{\mathrm{nwe}}$ \\
\hline Torques Shoulder & $\left\|\boldsymbol{\tau}_{\text {shoulder }}\right\|$ & $\varepsilon_{\mathrm{tsh}}$ \\
\hline Torques Lumbar & $\left\|\boldsymbol{\tau}_{\text {lumbar }}\right\|$ & $\varepsilon_{\text {tlb }}$ \\
\hline Back Flexion & $\left\|\theta_{L 5 S 1}^{Y}\right\|$ & $\varepsilon_{\text {back }}$
\end{tabular}

a set of movement demonstrations as Gaussian distributions [25]. The mean of those distributions are represented as a weighted sum of basis functions, $\phi_{t}$, defined at the learning of the ProMP. Therefore, a ProMP mean trajectory, $y_{t}^{t r a j}$, can be modulated by its weight vector, $\boldsymbol{w}_{t r a j}$ :

$$
y_{t}^{t r a j}=\phi_{t}^{\top} \boldsymbol{w}_{t r a j}
$$

Similarly to [26], all ProMP trajectories can be stacked into a single weight vector, that finally defines our parameters to be optimized: $\boldsymbol{w}=\left[\boldsymbol{w}_{1} \ldots \boldsymbol{w}_{n_{\text {trajs }}}\right]$.

The initial ProMP trajectories are a result of estimating the weights, $\boldsymbol{w}$, according to the initial movement demonstrations captured using a whole-body motion capture system.

\section{Trajectory Optimization}

We optimize a selection of the DHM body segment trajectories, $\boldsymbol{y}(\boldsymbol{w})$, through its optimizable parameters, $\boldsymbol{w}$, w.r.t. one of the ergonomic scores in table 1 with a single-objective optimizer, or with multiple scores, simultaneously, with a multiobjective optimizer. Given an episode $k$ in the optimization loop (Fig. 1), the point $\boldsymbol{w}^{k}$ is considered feasible if, and only if, the executions of the whole-body trajectories $\boldsymbol{y}\left(\boldsymbol{w}^{k}\right)$ respect some nonlinear constraints.

Trajectory Constraints: The DHM limbs and reference trajectories should always be within the environment workspace. That is, each ProMP weight is constrained to box boundaries that correspond to the DHM's reach in the workspace. Additionally, during the trajectories' execution, the DHM must never fall, and its hand(s) must reach all (activity-dependent) points of interest that are relevant for the activity. In order for the trajectory execution scores (1) to be comparable, the duration of every trajectory execution is always fixed for every episode. This trajectory optimization is, therefore, a derivativefree problem with black-box non-linear constraints.

Single-Objective Trajectory Optimization (SOTO): We bootstrap the optimization with the initial ProMP weights learned from the demonstration set. To optimize each one of the scores separately, we use single-objective optimization with the optimizer COBYLA (Constrained Optimization BY Linear Approximation) [27], a deterministic local optimizer that directly takes black-box constraints as inputs alongside any of the ergonomics scores accumulated by (1), and has already been used for constrained motion optimization problems [26]. The COBYLA implementation is taken from the C++ library NLopt [28].

Multi-Objective Trajectory Optimization (MOTO): To optimize for multiple scores at the same time, we advocate for multi-objective optimization. The goal becomes not to find 
one single optimal solution, but rather, a set of Pareto-optimal solutions that provide trade-off trajectories for conflicting ergonomics scores, i.e a Pareto front. By definition [9], within the Pareto front, all solutions are said to be dominant: given solutions $\boldsymbol{w}_{1}$ and $\boldsymbol{w}_{2}, \boldsymbol{w}_{1}$ is said to dominate $\boldsymbol{w}_{2}$ if and only if $\boldsymbol{w}_{1}$ provides better results for all objective functions; if one or more of $\boldsymbol{w}_{2}$ 's objectives is better than in $\boldsymbol{w}_{1}$, then both are dominant solutions with a trade-off between each other. We used the Non-dominated Sorting Genetic Algorithm II (NSGA II), a multi-objective evolutionary optimizer [9] implemented in the C++ library Sferes $_{v 2}$ [29].

Objective Function Penalties: Differently from COBYLA, NSGA-II's implementation does not handle specifying feasible/unfeasible points directly, so we modify the objective function (1) to penalize the unfeasible points. Each ergonomics score is penalized in case the DHM falls or it does not reach the activity's points of interest:

$$
\mathcal{J}_{o b j}=T_{f a l l} \mathcal{P}_{o b j}^{\text {fall }}+\mathcal{P}_{o b j}^{v i a}+\sum_{t \in[0 \ldots T]} \varepsilon_{o b j}^{2}(t)
$$

where $T_{\text {fall }} \in \mathbb{R}^{+}$is the period of time in which the DHM has been fallen, $\mathcal{P}_{o b j}^{\text {fall }} \in \mathbb{R}^{+}$is the fall penalty for a given score, and $\mathcal{P}_{o b j}^{\text {via }} \in \mathbb{R}^{+}$is the point-of-interest penalty for a given score. Each score is associated with a different penalty value, for they have different orders of magnitude.

Bootstrapping Initial Demonstrations: NSGA-II's implementation also does not allow defining initial trajectories directly. Hence, we modify the initial population sampling in order to bootstrap the human demonstrations. The $i$-th variable, $w_{i}$, of each initial individual is sampled using the initial ProMP $i$-th variable, $w_{i}^{\text {initial }}$ using a Gaussian distribution:

$$
w_{i}=\mathcal{N}\left(w_{i}^{\text {initial }}, \delta_{i} \beta\right)
$$

where $\delta_{i}$ is the largest distance between $w_{i}^{\text {initial }}$ and any of its box boundaries, and $\beta \in \mathbb{R}$ is a constant that modulates how much of the boundaries we want to sample initially. For instance, if $\beta=\frac{1}{3}$, then $p\left(w_{i}=\right.$ boundary $\left._{i}\right) \leq 0.3 \%$, i.e., we would sample the entire workspace with very low probabilities at each variable boundary. Here, we chose $\beta=\frac{1}{12}$, a low value, to keep the initial sample close to the initial demonstrations.

\section{EXPERIMENTS}

The proposed approach is used to optimize whole-body motions under a variety of body morphologies, ergonomics scores, and work activities. Two work activities commonly related to movements that are risky in terms of ergonomics are analyzed, A and B (Fig. 3), which are described hereafter.

Activity A - Pick and Place Object from a Shelf: The human has to reach an object located on a shelf with his/her right hand, take the object, and move it laterally toward the right side to another point on the same shelf. If the worker's shoulder level is below the shelf, this activity requires overhead work that could overload the worker's right shoulder.

To execute this activity, the DHM QP controller includes an additional task that commands the head to face the right hand. The task weights in the QP controller are set as: 1.0 for the feet position $(\mathrm{X}, \mathrm{Y}, \mathrm{Z})$, center of mass $(\mathrm{CoM})$ position $(\mathrm{X}, \mathrm{Y})$,

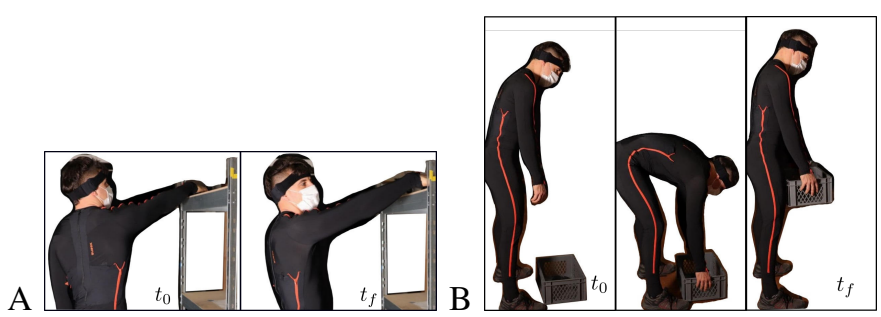

Fig. 3: Demonstrations for work activities A and B captured with the motion capture suit Xsens MVN. A: Pick and place a weight on a high shelf. B: Lift a box from the floor.

and hand position $(\mathrm{X}, \mathrm{Y}, \mathrm{Z}) ; 0.5$ for the hand orientation (roll, pitch, yaw); 0.1 for Pelvis position (Z), and Head orientation (roll, pitch, yaw); 0.05 for a reference body posture task; and 0.005 for a reference back lateral bending joint position task.

Activity B - Lift Box from the Floor: The human has to reach a box situated on the ground, in front of her/him, and with both hands, lift it to the waist level height. This activity commonly requires a great amount of effort surrounding the human's lumbar area, which could be overloaded in the case of excessive back flexion, and/or excessive manipulated weights.

To execute this activity, the weights in the QP controller are set as: 1.0 for the feet position $(\mathrm{X}, \mathrm{Y}, \mathrm{Z}), \mathrm{CoM}$ position $(\mathrm{X}, \mathrm{Y})$, hand position and orientation $(\mathrm{X}, \mathrm{Y}, \mathrm{Z}$, roll, pitch and yaw); 0.05 for the pelvis position and orientation ( $Z$, pitch), and reference body posture task; and 0.05 for reference joint positions at the ankles, knees, and back internal rotation and abduction joint positions.

\section{A. Experiment 1 - Effect of Varying Morphology}

The goal of this experiment is to show that optimal ergonomic motions are user-dependent. We generated 9 different DHM morphologies with 3 different body heights, and 3 different body mass indexes corresponding to underweight, average weight, and overweight morphologies (Tab. III). In this experiment, the right hand vertical position and the CoM ground projection trajectories were optimized for an activity A type of motion, in which the shelf is located at $1.5 \mathrm{~m}$ high and the start and end points for the hand are $30 \mathrm{~cm}$ apart. The initial hand trajectory was artificially generated as the minimum jerk trajectory between the start and end points. The hand trajectory was defined by a ProMP with 25 weights, and the CoM trajectory by a ProMP with 5 weights for each coordinate, $\mathrm{X}$ and $\mathrm{Y}$, therefore, $\boldsymbol{w} \in \mathbb{R}^{35}$.

For each morphology, we ran single-objective optimizations with 2 ergonomics scores for the shoulder: the RULA-C score (evaluates upper-body motions), and the shoulder torque score. The optimizer was set so that the optimization stopped after 1500 rollouts or when the improvement in cost function between successive rollouts was below $10^{-5}$.

\section{B. Experiment 2 - Effect of Ergonomics Scores}

The goal of this experiment is to show that SOTO with different ergonomics scores generates different optimal trajectories with possible negative impact on the overall ergonomics 
due to conflicting criteria. We optimize the motion for both types of activities, $\mathrm{A}$ and $\mathrm{B}$, and for each activity we run one SOTO for each ergonomics score listed in Tab. I] Differently from experiment 1 , here, the initial motion is captured from real human demonstrations (Fig. 3). In activity A, the shelf is located at $1.7 \mathrm{~m}$ high, and the start and end points are 0.64 $\mathrm{m}$ apart. The human demonstrator, as well as his DHM, are $1.85 \mathrm{~m}$ high, with $93 \mathrm{~kg}$, therefore, here, activity A required overshoulder work. For both activities, we instructed the human demonstrator to perfom a non-ergonomic demonstration (keeping hand above shoulder level in activity A and bending the back and not the knees in activity B), so that there was always a path for improvement in the optimization process. Additionally, weights of $1 \mathrm{~kg}$ were used for both activities to limit the risk of injuries. In the simulation, however, we used a $5 \mathrm{~kg}$ object (act. A) and a $10 \mathrm{~kg}$ box (act. B) to assess demanding tasks where the choice of postural strategies might have a larger impact on ergonomics scores.

In activity $\mathrm{A}$, the $\mathrm{CoM}(\mathrm{X}, \mathrm{Y})$, hand $(\mathrm{X}, \mathrm{Y}, \mathrm{Z})$, and Pelvis (Z) QP reference trajectories are optimized with 10, 30, and 10 ProMP weights respectively, totaling 50 parameters to be optimized. In activity B, the CoM (X,Y), and Pelvis (Z) QP reference trajectories are optimized with 10 , and 20 ProMP weights respectively, totaling 30 parameters to be optimized. For each parameterized trajectory, the initial values of the ProMP weights are learned from 5 human demonstrations. In both activities, the optimizer was set so that the optimization stopped after 1500 rollouts or when the improvement in cost function between successive rollouts was below $10^{-5}$.

\section{Experiment 3 - Multi-Objective Optimization}

In this experiment, our goal is to show that MOTO generates motions with better trade-offs between multiple ergonomics scores than SOTO. We ran the MOTO on the same activities as in experiment 2, including the same constraints and parameters for the DHM QP controller. Instead of including all the ergonomics scores in the optimization, we selected the scores that are most relevant for each activity. Activity A demands a significant motion from the right shoulder, and it is mainly an upper-body activity, so we chose to optimize the motion w.r.t torques shoulder, normalized whole-body effort, and RULA$\mathrm{C}$ scores. For activity B, both the shoulder and the lumbar joints are well demanded during the box lifting, so we chose to optimize the motion w.r.t. torques shoulder, and torques lumbar scores.

NSGA-II hyper-parameters are set as follows: cross rate = 0.5 ; population size $=100$; number of generations $=600$ (totalling 62000 rollouts per optimization execution). The mutation rates are set to 0.2 , and 0.4 for activities A and B respectively. Since NSGA-II is a stochastic algorithm, we ran the optimization, in parallel, 20 times.

\section{Results And Discussion}

\section{A. Experiment 1}

The optimization generated motions with improved ergonomics scores for each morphology with a median improvement of $16.9 \%$ and interquartile range (IQR) of $18.6 \%$ regarding the RULA-C score, as well as a median improvement
TABLE II: Improvement of the ergonomics score from the initial movement after SOTO for different morphologies.

\begin{tabular}{c|ccccc}
$m_{i}$ & Height (m) & B.M.I. & Weight $(\mathbf{k g})$ & $\mathcal{J}_{r c}$ & $\mathcal{J}_{\text {tsh }}$ \\
\hline \hline 1 & 2.0 & 18 & 72 & $8.3 \%$ & $29.3 \%$ \\
\hline 2 & 2.0 & 22 & 88 & $8.4 \%$ & $31.6 \%$ \\
\hline 3 & 2.0 & 30 & 120 & $8.1 \%$ & $29.0 \%$ \\
\hline \hline 4 & 1.8 & 18 & 58 & $16.9 \%$ & $26.1 \%$ \\
\hline 5 & 1.8 & 22 & 71 & $17.6 \%$ & $25.2 \%$ \\
\hline 6 & 1.8 & 30 & 97 & $14.3 \%$ & $21.4 \%$ \\
\hline \hline 7 & 1.6 & 18 & 46 & $33.5 \%$ & $19.1 \%$ \\
\hline 8 & 1.6 & 22 & 56 & $25.9 \%$ & $17.9 \%$ \\
\hline 9 & 1.6 & 30 & 77 & $28.0 \%$ & $16.8 \%$
\end{tabular}
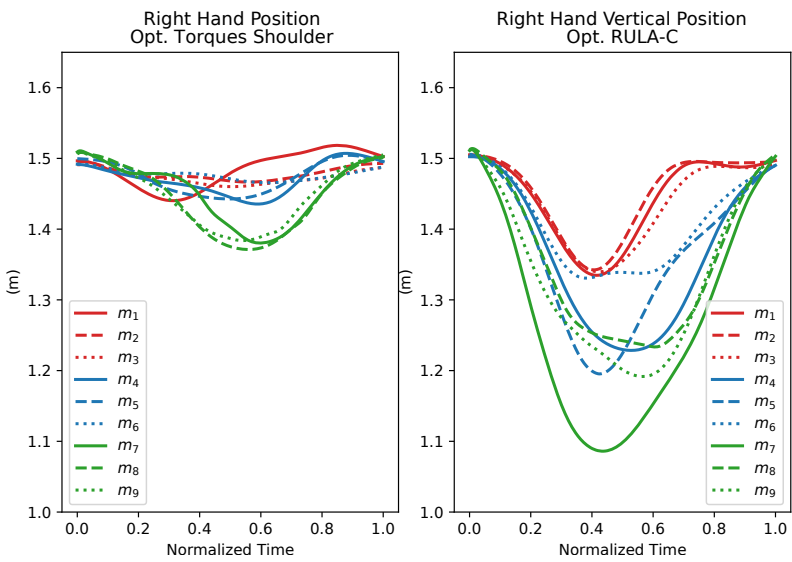

Fig. 4: Optimized hand trajectories w.r.t. torques shoulder and RULA-C scores for the morphologies $m_{i} \mid i \in[1 \ldots 9]$ in Tab. II in experiment 1. Tall in red, medium in blue, and short in green.

of $25.2 \%$ and IQR of $10.7 \%$ for the torque shoulder score (Tab. II] For both ergonomic scores, the hand trajectory of the short morphologies (m7, m8, m9) were distinguishingly lower than for the tall morphologies $(\mathrm{m} 1, \mathrm{~m} 2, \mathrm{~m} 3)$, which is consistent with reducing the arm elevation angle. For the tall morphologies and the torque shoulder score, the hand vertical trajectory did not deviate much from the initial trajectory (straight line at $1.5 \mathrm{~m}$ high). This was likely because the initial hand trajectory was already below the tall morphologies' shoulder level, and hence characterized a local minimum for this score. Indeed, lowering the arm even more would reduce the gravity torque at the shoulder, but it would require to move faster (since the task duration was fixed) thereby increasing the torque due to inertia. These results confirm that each individual needs to have a custom motion optimization for his/her body morphology.

\section{B. Experiment 2}

Each optimization improved the initial motion according to its ergonomics score (Fig. 5). In activity A, each optimization improved: back flexion by $99.37 \%$, RULA-C by $4.52 \%$, normalized whole-body efforts by $12.92 \%$, torques shoulder by $60.36 \%$, and torques lumbar by $77.24 \%$. In activity B, each optimization improved: back flexion by $93.42 \%$, RULA-C by $30.02 \%$, normalized whole-body efforts by $87.67 \%$, torques shoulder by $64.97 \%$, and torques lumbar by $67.32 \%$. 
Each one of the ergonomics scores have had a unique influence on the whole-body posture and efforts. For instance, the DHM's right elbow is more flexed during the optimal motion w.r.t. the torques shoulder score in comparison to the other motions during activity A (Fig. 6). This is likely due to the fact that flexing the elbow brings the arm closer to the torso, hence, decreasing the torques caused by gravity on the shoulder. During activity B, the initial motion has excessively high lumbar torques (Fig. 5) due to the large back flexion (Fig. 6). This motion strategy was penalized by all ergonomics scores, which in turn favored motions that reduce the back torque decreasing the DHM's back flexion, and increasing the DHM's knee flexion instead. Interestingly, this is the case even for the torque shoulder optimal motion, where the lumbar torque is not directly penalized, although with a lesser amount of knee flexion than the others.

The results confirm that solutions optimized for a given score may degrade other scores (Fig. 5). In activity A, minimizing the torque shoulder score increases the wholebody effort and back flexion, while in activity B, minimizing back flexion increases the torque shoulder score. Additionally, conflicting ergonomics scores could happen when optimizing for scores that do not evaluate the activity's main load requirements. For instance, in activity A, whose main load is at the shoulder, optimizing for back flexion highly increased torques at the shoulder, while optimizing for lumbar torques increased the whole-body efforts in comparison to the initial motion.

According to these results, optimizing for a single ergonomics score may not be advisable, and a more holistic approach concerning different ergonomics criteria must be sought for motion optimization.

\section{Experiment 3}

The Pareto front for both activities was computed for 20 MOTO replicates per activity (Fig. 7, and Fig. 8). The resulting Pareto fronts presented much starker score diversity between the Pareto-optimal solutions than the motions from the SOTO in experiment 2 (Fig. 5). This likely happened because NSGAII is a global optimizer, therefore, it explores the optimization space more efficiently than local optimizers. This diversity gives more options, and flexibility for the user to choose a Pareto-optimal solution according to given criteria.

To illustrate the advantage of using the MOTO approach, we visually selected some motions from each Pareto front of each activity with reasonable trade-offs between the scores (Fig. 7, Fig. 8), and compared them to the single objective solutions of the same scores (Table III). For activity A, $w_{A 3}^{\star}$ had similar elbow flexion trajectory to the SOTO w.r.t. torque shoulder score, as a matter of fact, this is a good solution if the user does not care about the generalized increase in the wholebody torques (indicated by $\mathcal{J}_{\text {nwe }}$ ). On the other hand, if both the whole-body torques and the torques at the shoulder are important for the user, $w_{A 4}^{\star}$ could be a more interesting choice. Similarly for activity $\mathrm{B}, w_{B 2}^{\star}$ is a movement that optimizes both shoulder and lumbar torques simultaneously, but if the user would prefer the minimum shoulder torques from the pareto front, then $w_{B 1}^{\star}$, with less knee flexion, would be a
TABLE III: Improvement of the ergonomics scores w.r.t. the initial motion after SOTO and MOTO. Worse performance in red. The multi-objective solutions are indicated in the Pareto fronts (Figs. 7 and 8 ).

(a) Activity A

\begin{tabular}{c|c|c|c} 
Motion & $\mathcal{J}_{\text {tsh }}$ & $\mathcal{J}_{\text {nwe }}$ & $\mathcal{J}_{\text {rc }}$ \\
\hline Initial & $100 \%$ & $100 \%$ & $100 \%$ \\
\hline Single Obj. $\mathcal{J}_{\text {tsh }}$ & $39.4 \%$ & $146.2 \%$ & $105.7 \%$ \\
\hline Single Obj. $\mathcal{J}_{\text {nwe }}$ & $99.1 \%$ & $80.2 \%$ & $102.4 \%$ \\
\hline Single Obj. $\mathcal{J}_{r c}$ & $77.6 \%$ & $90.8 \%$ & $95.5 \%$ \\
\hline Multi-Obj. $w_{A 1}^{\star}$ & $83.9 \%$ & $115.9 \%$ & $93.4 \%$ \\
\hline Multi-Obj. $w_{A 2}^{\star}$ & $72.9 \%$ & $41.2 \%$ & $102.7 \%$ \\
\hline Multi-Obj. $w_{A 3}^{\star}$ & $50.1 \%$ & $198.0 \%$ & $100.5 \%$ \\
\hline Multi-Obj. $w_{A 4}^{\star}$ & $35.8 \%$ & $53.4 \%$ & $97.0 \%$
\end{tabular}

(b) Activity B

\begin{tabular}{c|c|c} 
Motion & $\mathcal{J}_{t s h}$ & $\mathcal{J}_{t l b}$ \\
\hline Initial & $100 \%$ & $100 \%$ \\
\hline Single Obj. $\mathcal{J}_{t s h}$ & $33.6 \%$ & $70.6 \%$ \\
\hline Single Obj. $\mathcal{J}_{t l b}$ & $78.8 \%$ & $32.8 \%$ \\
\hline Multi-Obj. $w_{B 1}^{\star}$ & $16.0 \%$ & $36.9 \%$ \\
\hline Multi-Obj. $w_{B 2}^{\star}$ & $22.4 \%$ & $26.0 \%$ \\
\hline Multi-Obj. $w_{B 3}^{\star}$ & $73.4 \%$ & $24.4 \%$
\end{tabular}

better choice. Note that $w_{B 1}^{\star}$, also has a greater reduction on the lumbar torques than the SOTO solution for the shoulder torques. Additionally, most solutions from the Pareto fronts have improved their ergonomics scores, even for scores that were not being optimized (Fig. 6). This is likely due to those scores not being in conflict with the optimized ones.

Video: To show that Pareto-optimal solutions obtained by MOTO are better ergonomics trade-offs than those obtained by SOTO, we refer the reader to the video attachment where we compare the different whole-body movements executed by our DHM. Clearly, optimizing for a single criteria easily produces unrealistic movements that one could actually refer to as non ergonomic: e.g., we point out the solution in activity A that minimizes the lumbar torques with a very awkward non-ergonomic motion. Movements generated by our MOTO approach are more plausible and ergonomically adequate.

In conclusion, generating whole-body motion with MOTO provides better trade-offs among multiple ergonomics criteria; and because many solutions are generated, we obtain a tool that enables a user (i.e., an ergonomist) to choose from a set of ergonomic motions that are often better than the ones generated with SOTO.

\section{CONCLUSION}

We showed that single-objective optimization may not be sufficient to obtain satisfactory ergonomic motions, since optimizing for only one criterion often produces motions that are less ergonomic w.r.t. other criteria. Instead, in our approach we generate a set of Pareto-optimal motions with respect to multiple ergonomic scores. This allows us to simultaneously consider several criteria without requiring an ergonomics expert to preset weights for the different criteria prior to the optimization, as would be the case if the criteria were aggregated in a weighted sum. With our approach, solutions corresponding to any specific trade-off between the different ergonomic scores can be selected afterwards to match, for 

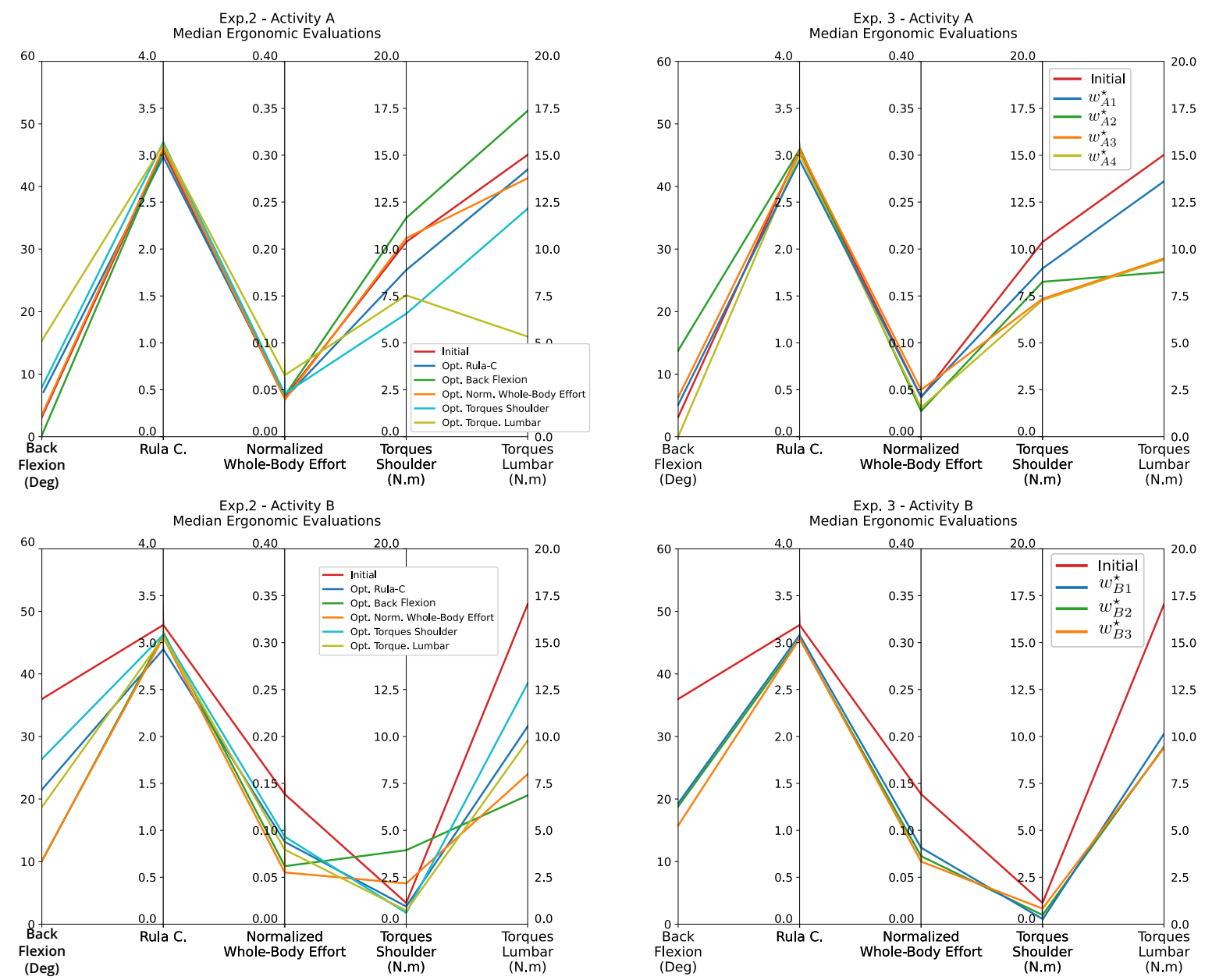

Fig. 5: Experiment 2 (SOTO) and Experiment 3 (MOTO) - The median of the ergonomics scores during the execution of the initial and optimal motions. Lines of the same color represent one motion, and each axis represents one of the ergonomics scores. The motions in experiment 2 are taken from 5 independent single-objective optimizations for each activity. The motions in experiment 3 are taken from the respective Pareto fronts for each activity (Fig. 7 and 8 ).
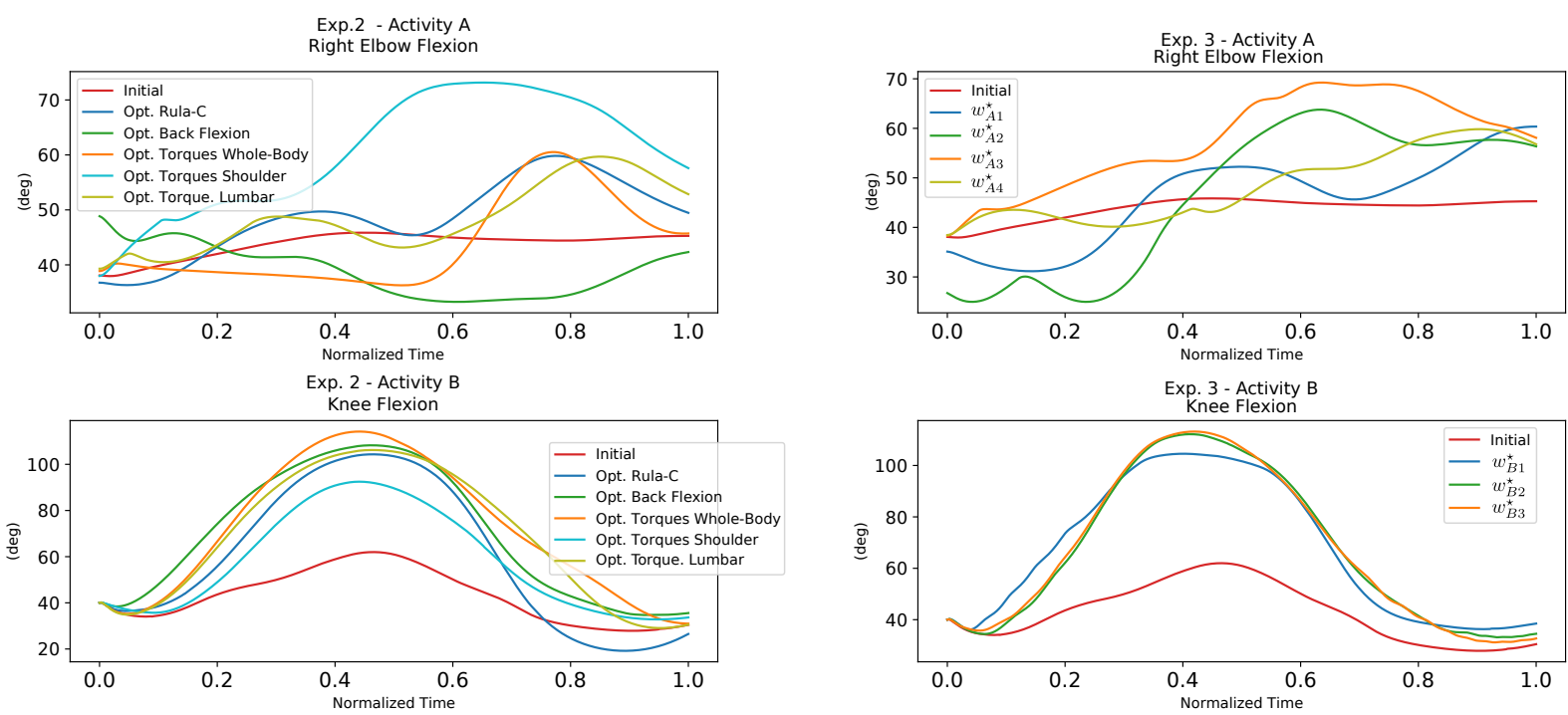

Fig. 6: Experiment 2 (SOTO) and Experiment 3 (MOTO) - Time evolution of selected angles of the initial movement and the optimized motions: elbow flexion for activity A (Reaching); knee flexion for activity B (Lifting). 


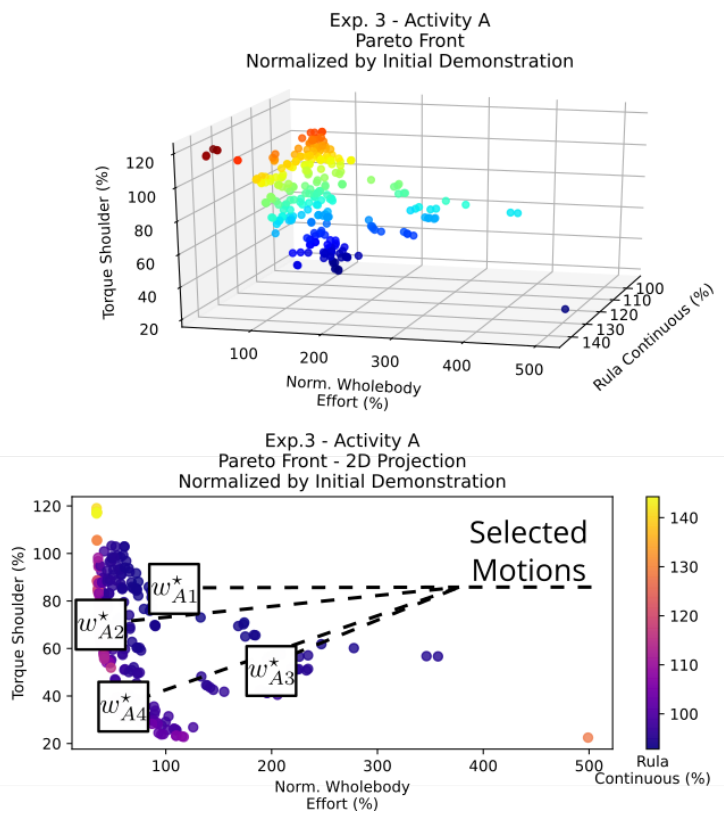

Fig. 7: Experiment 3 - Activity A - Pareto front. The scores values are normalized by those of the initial motion. The bottom image is a $2 \mathrm{D}$ projection of the $3 \mathrm{D}$ Pareto front, the third objective is represented by a color scale on each point.

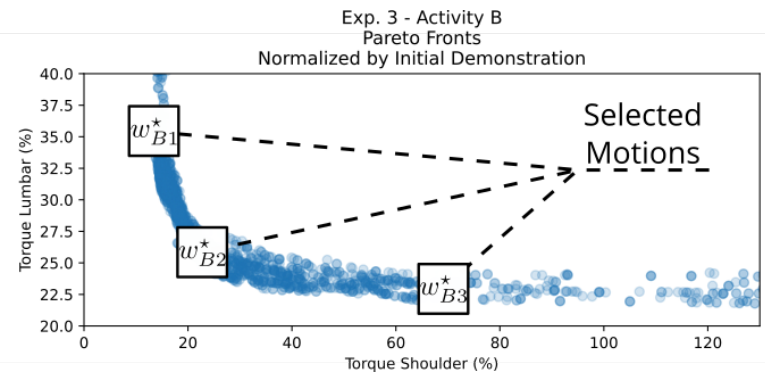

Fig. 8: Experiment 3 - Activity B - Pareto front. The scores values are normalized by those of the initial motion.

instance, the user preferences, or medical condition or the recommendations of an ergonomist. But importantly, this selection does not require to re-run the optimization, which represents a significant gain of time. Preliminary discussions with occupational ergonomists confirmed the potential of our tool, provided that we include learning implicit preferences for the Pareto-optimal solutions. This will be object of future work, possibly using preference learning algorithms [30].

Additionally, our approach has direct applications in humanrobot physical interaction: a collaborative robot could be used to drive the human user towards an ergonomic posture when performing a joint task. For example, from the output of our optimization we can easily extract a user- and activity-specific optimal human hand trajectory, and then use it as the reference end-effector trajectory during a collaborative task.

\section{REFERENCES}

[1] L. Punnett et al., "Work-related musculoskeletal disorders: the epidemiologic evidence and the debate," J. Electromyogr. Kinesiol., 2004.

[2] K. K. Lett and S. M. McGill, "Pushing and pulling: personal mechanics influence spine loads," Ergonomics, 2006.
[3] B. Busch et al., "Postural optimization for an ergonomic human-robot interaction," in IROS, 2017.

[4] W. Kim et al., "Anticipatory robot assistance for the prevention of human static joint overloading in human-robot collaboration," IEEE RA$L, 2018$.

[5] A. G. Marin et al., "Optimizing contextual ergonomics models in humanrobot interaction," in IEEE IROS, 2018.

[6] A. Shafti et al., "Real-time robot-assisted ergonomics," in IEEE ICRA, 2019.

[7] L. Vianello et al., "Human posture prediction during physical humanrobot interaction," IEEE RA-L, 2021.

[8] B. Busch, M. Toussaint, and M. Lopes, "Planning ergonomic sequences of actions in human-robot interaction," in ICRA, 2018.

[9] K. Deb, "Multi-objective optimisation using evolutionary algorithms: an introduction," in Multi-objective evolutionary optimisation for product design and manufacturing. Springer, 2011, pp. 3-34.

[10] P. Maurice et al., "Assessing and improving human movements using sensitivity analysis and digital human simulation," Int. J. of Comput. Integr. Manuf., 2019.

[11] S. Scataglini and G. Paul, DHM and Posturography. Elsevier, 2019.

[12] L. v. der Spaa et al., "Predicting and optimizing ergonomics in physical human-robot cooperation tasks," in IEEE ICRA, 2020.

[13] R. Rahal et al., "Caring about the human operator: Haptic shared control for enhanced user comfort in robotic telemanipulation," IEEE Trans. on Haptics, 2020.

[14] A. Yazdani and R. S. Novin, "Posture estimation and optimization in ergonomically intelligent teleoperation systems," HRI, 2021.

[15] Y. Xiang et al., "Human lifting simulation using a multi-objective optimization approach," Multibody System Dynamics, 2010.

[16] I. Pascual et al., "Implementation of ergonomics evaluation methods in a multi-objective optimization framework," in $D H M, 2020$.

[17] P. Maurice et al., "Human-oriented design of collaborative robots," Int. J. of Ind. Ergonomics, 2017.

[18] L. F. C. Figueredo et al., "Human comfortability: Integrating ergonomics and muscular-informed metrics for manipulability analysis during human-robot collaboration," IEEE RA-L, 2021.

[19] D. Chaffin et al., Occupational Biomechanics. Wiley, 2006.

[20] Open Design Lab, Penn State Univ., "Open design lab scaling calculator," http://tools.openlab.psu.edu/tools/proportionality_constants.htm

[21] J. Lee et al., "DART: Dynamic animation and robotics toolkit," Journal of Open Source Software, 2018.

[22] E. Mingo Hoffman et al., "Robot control for dummies: Insights and examples using opensot," in Humanoids, 2017.

[23] G. David, "Ergonomic methods for assessing exposure to risk factors for work-related musculoskeletal disorders," Occup. medicine, 2005.

[24] L. McAtamney and N. Corlett, "Rula: a survey method for the investigation of work-related upper limb disorders," Applied Ergonomics, 1993.

[25] A. Paraschos, C. Daniel, J. Peters, and G. Neumann, "Using probabilistic movement primitives in robotics," Autonomous Robots, 2018.

[26] W. Gomes et al., "Humanoid whole-body movement optimization from retargeted human motions," in Humanoids, 2019.

[27] M. J. Powell, "A direct search optimization method that models the objective and constraint functions by linear interpolation," in $A d v$. in Opt. and Num. analysis. Springer, 1994.

[28] S. G. Johnson, The NLopt nonlinear-optimization package. [Online]. Available: http://ab-initio.mit.edu/nlopt

[29] J.-B. Mouret and S. Doncieux, "SFERESv2: Evolvin' in the multi-core world," in Proc. of Congress on Evolutionary Computation (CEC), 2010.

[30] K. Taylor et al., "Bayesian preference learning for interactive multiobjective optimisation," in ACM, ser. GECCO, 2021. 